\title{
Facile one-step photopatterning of polystyrene films
}

\author{
Takashi Ubukata, Yusuke Moriya and Yasushi Yokoyama \\ The development of nano- and micropatterned materials is a key component of advanced nanotechnology. We report herein, \\ a photo-based method for patterning general-purpose polymer films. Patterned ultraviolet (UV) light irradiation of a thin \\ polystyrene film under controlled temperature generates surface relief structures by mass transfer from shaded to irradiated \\ areas. The resulting surface relief structure can be arbitrarily erased by heating and reconstructed by patterned UV light \\ irradiation, and this process is repeatable. This new method thus enables fabrication of dynamic reversible patterning \\ structures from various general polymers.
}

Polymer Journal (2012) 44, 966-972; doi:10.1038/pj.2012.40; published online 28 March 2012

Keywords: mass transfer; photopatterning; polystyrene; surface relief; thin film

\section{INTRODUCTION}

Since the past four decades, photolithography has been an important method for forming patterning structures on photoresponsive films. With increasing miniaturization, device density has increased. ${ }^{1,2}$ Several patterning methods exist today, making photolithography a versatile tool. ${ }^{3,4}$

Among existing patterning methods, the photoinduced surface relief grating (SRG) formation on films containing azobenzene chromophore is both attractive and novel, as documented in a recent book $^{5}$ and reviews. ${ }^{6-10}$ The key to SRG formation is thought to be repeatable trans-cis-trans isomerization cycling and reorientation of the azobenzene chromophore upon irradiation with polarized visible (vis) light, typically from the 488- or 514-nm lines of an argon ion laser. SRGs are fabricated by mass transport when an azobenzenecontaining film is irradiated with spatially patterned light at temperatures below the film's glass transition temperature $\left(T_{\mathrm{g}}\right)$.

On films containing azobenzene chromophore, photoinduced SRG formation by this method has significant technological advantages because it involves just a facile, entirely optical single step with no wet chemistry. In addition, SRGs can be erased using uniform light or heating above $T_{\mathrm{g}}{ }^{11}$ and then reconstructed, suggesting possible applications such as for active optical devices and memories. ${ }^{6,9,12-14}$

However, this method also has a significant disadvantage: its use of azobenzene compounds as optical elements. Such use is undesirable because both the trans and cis isomers of push-pull-type azobenzene compounds, which are typically used to form SRG materials, have strong absorption bands in the vis region. A sophisticated solution has been reported that involves the formation of colorless SRGs by the use of the SRG-forming materials with detachable azobenzene units. ${ }^{15,16}$ Another novel solution has also been reported that involves photoinduced SRG formation on films containing photochromic compounds other than azobenzenes in which one of the two stable isomers is colorless. ${ }^{17-20}$ Finally, a solution of particular interest has been reported, in which SRGs on amorphous molecular films of photochromic spirooxazine were shown to be erasable by heating. Furthermore, these films could be reversibly constructed by spatially patterned light irradiation without a significant photo or thermal degradation. ${ }^{19}$ However, this last system is unstable against self-crystallization and mechanical stress because the amorphous film is composed of low molecular weight molecules.

In this study, we investigated reversible SRG formation using general purpose polymer films containing no photochromic agent. To our knowledge, this is the first time that such a strategy has been considered. Several publications are devoted to simple methods of fabrication of surface relief structures involving systems that do not need photoisomerization. ${ }^{21-48}$ However, the majority of these methods involve photoablation, ${ }^{21-26}$ photopolymerization ${ }^{27-29}$ or photocrosslinking. ${ }^{30-44}$ Hence, reversible SRG formation has not been reported in these systems.

For the general purpose polymer in our investigation, we selected polystyrene (PS). Among its advantages, it is ubiquitous and commercially available. Moreover, a PS-based system not only avoids the problems associated with azobenzene-functionalized compounds but also does not contain any additional dyes and is thus transparent to vis light, greatly expanding the range of possible applications. An encouraging related fact is that even pure PS films produce reversible SRGs, suggesting that photoisomerization of the chromophore is not always essential for lateral mass transfer.

\section{EXPERIMENTAL PROCEDURE}

\section{Materials}

Monodisperse PS samples were purchased from Shodex, Tokyo, Japan $\left(M_{\mathrm{w}}=\right.$ $2.97 \times 10^{3}, M_{\mathrm{w}} / M_{\mathrm{n}}=1.05, M_{\mathrm{w}}=4.49 \times 10^{3}, M_{\mathrm{w}} / M_{\mathrm{n}}=1.04, M_{\mathrm{w}}=1.07 \times 10^{4}$, 
$M_{\mathrm{w}} / M_{\mathrm{n}}=1.02$ and $\left.M_{\mathrm{w}}=1.98 \times 10^{4}, M_{\mathrm{w}} / M_{\mathrm{n}}=1.02\right)$, Alfa Aesar, Ward Hill, MA, USA $\left(M_{\mathrm{w}}=3.35 \times 10^{3}, M_{\mathrm{w}} / M_{\mathrm{n}}=1.10\right)$ and Chemco Scientific Co., Ltd., Osaka, Japan $\left(M_{\mathrm{w}}=6.5 \times 10^{4}, M_{\mathrm{w}} / M_{\mathrm{n}}<1.06\right)$ and used without further purification. Chloroform for the film preparation was of a spectroscopic grade (Wako Pure Chemical Industries, Ltd., Osaka, Japan).

\section{Film preparation}

A thin PS film was prepared by spin coating from a chloroform solution of PS (typically $1.1 \mathrm{wt} \%$ ) on a clean glass, quartz or $\mathrm{CaF}_{2}$ substrate. A spincoater (Mikasa 1H-D7, Mikasa Co. Ltd., Tokyo, Japan) was used with two program steps (step 1: 1000 r.p.m., 50 s; step 2: 3000 r.p.m., 2 s). Film thickness was calculated by curve fitting the reflection fringe obtained from a UV-vis-nearinfrared (IR) spectrometer (Jasco V-670, Jasco Corporation, Hachioji, Japan) equipped with an absolute reflectance measurement unit (ARSN-733). Typical thicknesses were in the range $160-180 \mathrm{~nm}$.

\section{Photoirradiation}

A high-pressure mercury lamp (Ushio SX-UI 500HQ, Ushio Inc., Tokyo, Japan) was used as a UV-light source. Light from the lamp was passed through a water filter $(5 \mathrm{~cm})$ and a long-pass filter (UV29). It was then collimated to obtain a homogeneous light intensity of $175 \mathrm{~mW} \mathrm{~cm}^{-2}$, evaluated at a wavelength of $500 \mathrm{~nm}$ using a power meter (Newport 1830-C, Newport Corporation, Irvine, CA, USA) equipped with a detector (818-UV) over the entire irradiated area. Light intensity was measured in this manner for two reasons: an emission line to produce SRG could not be separated and experiments of SRG production were performed using some types of longpass filters. To estimate UV intensity more accurately, the intensity of light through a water filter, UV29, and a bandpass filter, UVD33S, was measured. By superimposing UVD33S, the UV intensity was decreased by $78-71 \%$, depending on the detection wavelength from 277 to $366 \mathrm{~nm}$. Considering the transmittance of UVD33S in the UV region, the UV intensity used in the experiments $\left(175 \mathrm{~mW} \mathrm{~cm}^{-2}\right.$ at $\left.500 \mathrm{~nm}\right)$ corresponds to the UV intensity from 46 to $57 \mathrm{~mW} \mathrm{~cm}^{-2}$ at 277 and $365 \mathrm{~nm}$. However, this value was overesitmated because the UV light includes ineffective emission lines such as 313, 334 and $366 \mathrm{~nm}$ for SRG production. Patterned light irradiation was performed using the light source described above through a custom-made photomask (Toppan Printing Co., Ltd., Tokyo, Japan $)^{49}$ attached to the PS film. A gap of $\sim 1 \mu \mathrm{m}$ was produced between the photomask and the film surface by a bump in the film at the edge of the substrate. During irradiation, the film was set on a hotplate (Corning PC-420D, Corning Incorporated, New York, NY, USA) and the film temperature was measured using an IR thermometer (Horiba IT-550, Horiba Ltd., Kyoto, Japan). After irradiation, the film was immediately quenched to room temperature.

\section{Physical measurements}

UV-vis spectra were recorded on a UV-vis spectrometer (Shimadzu Multispec1500, Shimadzu Corporation, Kyoto, Japan) to confirm the film quality. Surface relief structures were observed with an atomic force microscope (AFM; Shimadzu 9500 (Shimadzu Corporation) equipped with an Olympus OMCLTR800PSA-1 (Olympus Corporation, Tokyo, Japan) cantilever or SII SPA300/ SPI3700 (Seiko Instruments Inc., Chiba, Japan) equipped with a SN-AF1 cantilever) in the contact mode. Relief height is defined as the difference in the vertical distance between the central part of the irradiated and shaded areas. Values of $T_{\mathrm{g}}$ for each PS sample in the bulk were obtained with a differential scanning calorimeter (Seiko Instruments DSC6100, Seiko Instruments Inc.) equipped with an instrument controller (Extra6000) at a heating/cooling rate of $10 \mathrm{~K} \mathrm{~min}^{-1}$. Changes in PS thermal properties upon UV light irradiation were measured using the cast PS film in an aluminum pan at heating and cooling rates of $5 \mathrm{~K} \mathrm{~min}^{-1}$ and $20 \mathrm{~K} \mathrm{~min}^{-1}$, respectively. PS molecular weights after irradiation were determined using a gel permeation chromatograph (Shimadzu CBM-20 A/LC20AD, Shimadzu Corporation) equipped with a gel permeation chromatograph-802C/803C column in CTO-20 A (313 K) and a UV-light detector (SPD-10 A, $262 \mathrm{~nm}$ ). Samples were dissolved in chloroform, which was used as the moving phase (flow rate $0.5 \mathrm{ml} \mathrm{min}^{-1}$ ). IR spectra were recorded using a Fourier transform IR spectrometer (Jasco FT/IR-4100, Jasco Corporation).

\section{RESULTS AND DISCUSSION}

\section{SRG topology on PS film}

Figure 1 shows AFM images of the surface relief structures of PS films after UV light irradiation through various photomask patterns. Irradiation through a grating photomask produces a regular sinusoidal surface modulation (Figure 1a). Its spatial period, $8 \mu \mathrm{m}$, is coincident with that of the photomask, and the observed modulation depth is $>200 \mathrm{~nm}$, larger than the initial film thickness of $170 \mathrm{~nm}$. The photomodulated structure remains stable and unchanged for at least 14 months at room temperature. When the patterned UV light irradiation was performed using the grating photomask with different periods, modulation depth changed depending on the periods (Supplementary Figure S1).

To investigate the direction of material transfer, we performed an additional experiment, irradiation through a single-slit photomask (Figure $1 \mathrm{~b}$; slit width $4.0 \mu \mathrm{m}$ ). The cross-sectional topography shows the top of the convex irradiated area to be higher and the base of the depression to be lower than the initial surface level. Thus, lateral material transfer clearly occurs from the shaded areas to the irradiated areas, enabling the formation of variable surface structures (Figures $1 \mathrm{c}$ and $\mathrm{d}$ ).

To induce the formation of surface relief structures, PS films must be irradiated with photons of sufficient energy. We therefore performed irradiation by passing light from a high-pressure mercury lamp (Ushio Inc. SX-UI 500HQ) through a 5-cm water filter to eliminate IR radiation, and then through various long-pass filters (UV27, 29, 31, 35 and Pyrex glass). Long-wavelength light provided by the UV31, UV35 and Pyrex glass filters did not induce any surface relief structures on the film. In contrast, short-wavelength light provided by the UV27 and UV29 filters induced sufficient SRGs. For the UV27 filter, the cross-sections of the induced SRGs frequently showed scraped features at the top of the convex center of the irradiated region (Supplementary Figure S2), resulting in a lower relief height $(10 \mathrm{~nm})$ than that produced by the UV29 filter $(80 \mathrm{~nm})$ under the same conditions $\left(175 \mathrm{~mW} \mathrm{~cm}^{-2}\right.$, $90 \mathrm{~min}, 368 \mathrm{~K})$. We presume that the scraped features are caused by ablation.

These results indicate that deep-UV light of wavelength $<300 \mathrm{~nm}$, sufficient to excite the electronic state of PS, is essential for inducing SRGs on PS films. However, light of much shorter wavelength, such as $254 \mathrm{~nm}$, tends to induce ablation by producing volatiles at the irradiated area, ${ }^{50-57}$ and hence may decrease SRG formation. Therefore, we performed further irradiation experiments by passing light from a high-pressure mercury lamp through a water filter and then through a long-pass filter (UV29).

\section{Temperature dependence of SRG topology}

The efficiency of PS transfer strongly depends on the temperature of the film during spatially patterned UV light irradiation. Figure 2 shows plots of relief height as a function of film temperature for PS films of various molecular weights and accordingly different values of $T_{\mathrm{g}}$. For the PS film of $M_{w}=2.97 \times 10^{3}$, film height increases gradually at $T=351 \mathrm{~K}\left(4 \mathrm{~K}\right.$ higher than the bulk value of $\left.T_{\mathrm{g}}\right)$ and reaches a maximum at $\sim 368 \mathrm{~K}\left(21 \mathrm{~K}\right.$ higher than the bulk value of $\left.T_{\mathrm{g}}\right)$. The various relief heights follow an approximately Gaussian distribution centered at this maximum temperature. For the PS films of different molecular weights, film height behaves similarly, showing maximum mass transfer efficiency $\sim 20 \mathrm{~K}$ higher than the associated $T_{\mathrm{g}}$ value. This temperature dependence is probably due to competition between increasing molecular mobility and the smoothing effect of surface tension $^{58,59}$ with increasing temperature. ${ }^{60}$ The PS films with much 
a
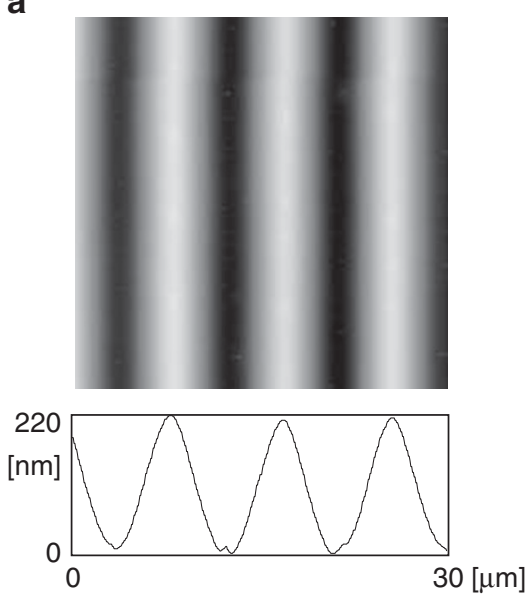

C

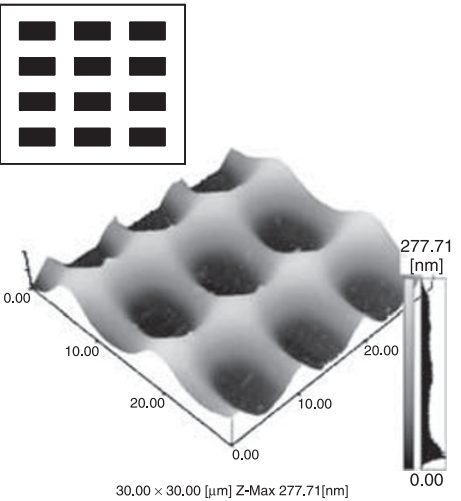

b
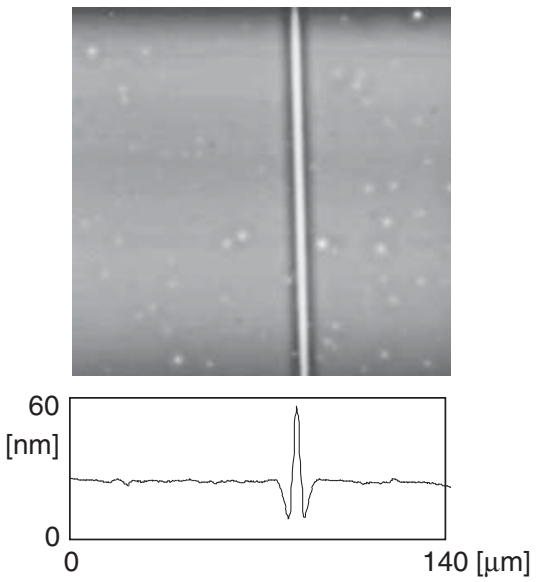

d

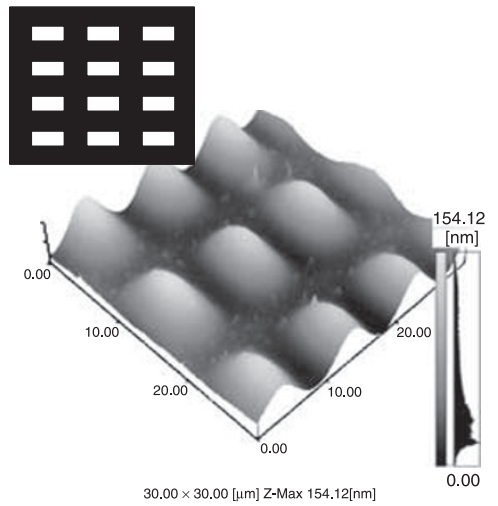

Figure 1 AFM images of the surface relief structures in PS (various $M_{\mathrm{w}}$ values) films of various thicknesses after UV light irradiation for 90 min at various temperatures through various photomask patterns: (a) $M_{\mathrm{w}}=4.49 \times 10^{3}, 170 \mathrm{~nm}, 381 \mathrm{~K}$, pattern of stripes with a period of $8 \mu \mathrm{m}$; (b) $M_{\mathrm{w}}=2.97 \times 10^{3}$, $54 \mathrm{~nm}, 368 \mathrm{~K}$, pattern with a single $4.0-\mu \mathrm{m}$-wide slit; (c) $M_{\mathrm{w}}=4.49 \times 10^{3}, 170 \mathrm{~nm}, 384 \mathrm{~K}$, pattern of rectangles with dimensions of $4 \times 8 \mu \mathrm{m}^{2}$ separated by $4 \mu \mathrm{m}$ and (d) $M_{\mathrm{w}}=4.49 \times 10^{3}, 170 \mathrm{~nm}, 384 \mathrm{~K}$, the inverted image of (c). A full color version of this figure is available at Polymer Journal online.

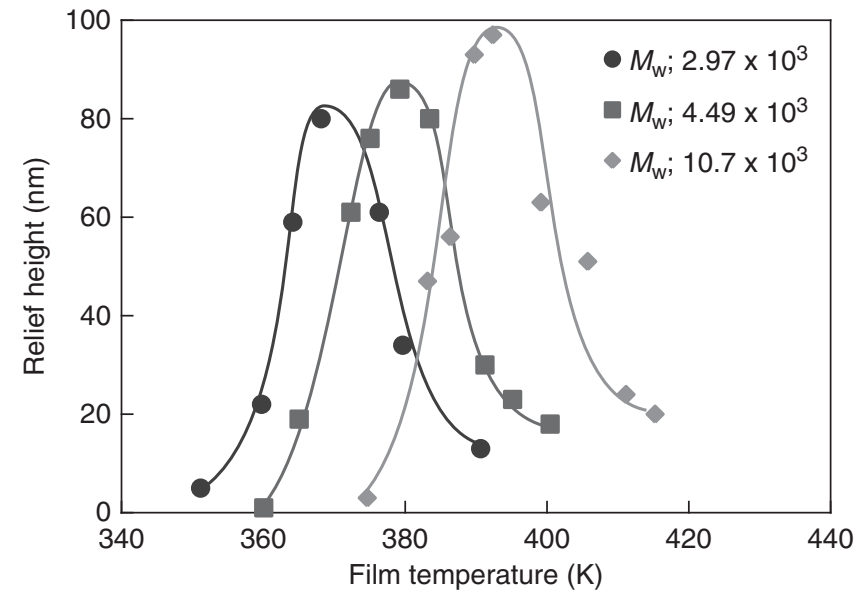

Figure 2 Plots of relief height as a function of film (thickness $180 \mathrm{~nm}$ ) temperature for three PS films (circles: $M_{\mathrm{w}}=2.97 \times 10^{3}, T_{\mathrm{g}}=347 \mathrm{~K}$; squares: $M_{\mathrm{w}}=4.49 \times 10^{3}, \quad T_{\mathrm{g}}=359 \mathrm{~K}$ and diamonds: $M_{\mathrm{w}}=10.7 \times 10^{3}$, $T_{\mathrm{g}}=369 \mathrm{~K}$ ). The films were measured after UV light irradiation through a photomask (stripes with a period of $5 \mu \mathrm{m}$ ) for $90 \mathrm{~min}$. A full color version of this figure is available at Polymer Journal online.

higher $M_{\mathrm{w}}$ also exhibited similar tendency regarding temperature dependence, and efficient maximum mass transfer was observed at $\sim 40 \mathrm{~K}$ higher than the associated $T_{\mathrm{g}}$ value (Supplementary Figure
S3). Thus, it is demonstrated that this SRG formation method can be applied to PS films of various molecular weights.

\section{Irradiation-time dependence of SRG morphology}

To investigate the process of mass transfer, we examined, by AFM, surface relief structures produced with different irradiation times. Figure 3 shows plots of relief height as a function of irradiation time for PS films. Initially, the surface relief structure grows slowly, probably because relief formation requires an induction period. After irradiation for $90 \mathrm{~min}$, the cross-sectional morphology remains stably sinusoidal and the relief height reaches a maximum of $220 \mathrm{~nm}$. After irradiation for $>120 \mathrm{~min}$, the cross-sectional morphology of each convex surface relief structure changes into two split peaks with a small trench, which resembles SRG produced by long-pass filter UV27 rather than UV29 (Supplementary Figure S2a), and the relief height decreases gradually as the trench grows deeper (Supplementary Figures S4a and b). These results suggest that photoreaction, including ablation, occurs preferentially at the center of persistently irradiated regions because of the gradation amount of lateral transfer of fresh PS molecules. In fact, we observed an obvious decrease in thickness after prolonged uniform UV light irradiation of the PS film under the same conditions as those used for fabrication of the surface relief structure (23\% decrease in thickness after irradiation for $90 \mathrm{~min}$ ) (Supplementary Figure S5). Significantly, the flow of lateral material transfer from shaded to irradiated areas exceeds the amount of ablation at the irradiated areas. Hence, a convex structure emerges at the irradiated areas. 


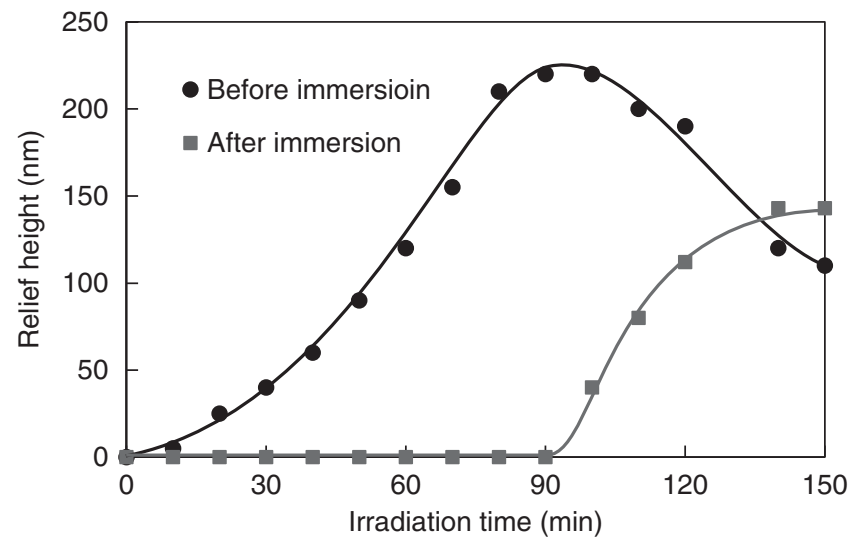

Figure 3 Plots of relief height as a function of irradiation time for PS $\left(M_{\mathrm{w}}=3.35 \times 10^{3}\right)$ films (thickness $160 \mathrm{~nm}$ ). The film was measured at two stages: just after UV light irradiation through a photomask (stripes with a period of $8 \mu \mathrm{m}$ ) at $379 \mathrm{~K}$ (circles) and after overnight immersion in chloroform (squares). A full color version of this figure is available at Polymer Journal online.

\section{Mechanism of SRG formation}

To further investigate the irradiated area, we irradiated the thin PS film uniformly under the same conditions as those used for fabrication of the surface relief structure. Figure 4 shows UV-vis absorption spectra of the PS film. After irradiation for $90 \mathrm{~min}$ (dashed line), absorbance $>225 \mathrm{~nm}$ is markedly higher than that before irradiation (solid line), indicating modification of the PS molecule by a ketone group and generation of an unsaturated chain. ${ }^{54}$ After immersion overnight in chloroform (dotted line), which is a good solvent for PS, most of the film dissolves.

Figure 5 shows gel permeation chromatograph plots for solutions from a specimen after different irradiation times, normalized to the peak intensity of the original PS molecule observed after a retention time of $29.8 \mathrm{~min}$. As irradiation time increases, components with retention times shorter than that of the original PS peak appear and increase. This result indicates that dimerization and further oligomerization of the PS molecule occur upon irradiation. In addition, simultaneous fragmentation of the PS molecule occurs at longer retention times than for the original PS peak. Such photoreactions were accelerated when the PS film was irradiated with deep-UV light including wavelength of $<300 \mathrm{~nm}$ at elevated temperature. (Supplementary Figure S6). Thus, it is confirmed that the generation of SRG is closely related to the photoreaction of PS. Among the numerous mechanisms for the photoreaction of PS, following reactions are accepted as part of photoreactions of solid PS in air. $^{61-64}$ When irradiated with deep-UV light, PS forms a PS radical, which leads to a peroxy radical and eventually PS hydroperoxide. The latter photolyzes into an alkoxy radical, leading to chain scission with the formation of a shorter chain PS radical and carbonyl species. In fact, our Fourier transform-IR spectra show the generation of carbonyl and hydroxy groups (Supplementary Figure S7), indicating that degradative photooxidation occurs during photoirradiation. ${ }^{65-66}$ Thus, we assume that PS-related radicals such as PS and alkoxy radicals might recombine to produce dimers and further oligomers.

We immersed formed SRGs overnight in chloroform and observed the surface of the immersed substrates by AFM. A relief structure prepared by irradiation for $90 \mathrm{~min}$ is completely washed out and only the flat substrate remains. However, relief structures prepared by

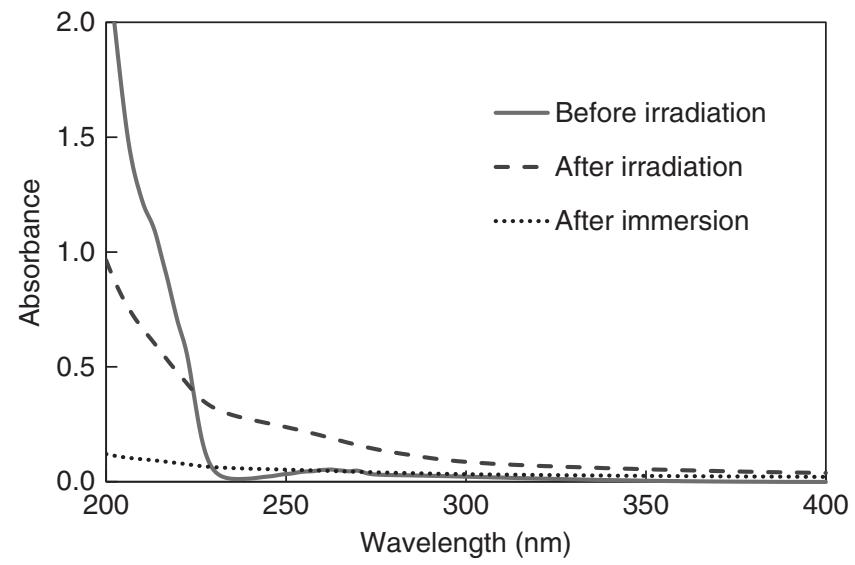

Figure 4 UV-vis absorption spectra of a PS $\left(M_{w}=3.35 \times 10^{3}\right)$ film (thickness $160 \mathrm{~nm}$ ). The film was measured at three stages: before UV light irradiation (solid line), after UV light irradiation for $90 \mathrm{~min}$ at $379 \mathrm{~K}$ (dashed line) and after overnight immersion in chloroform (dotted line). A full color version of this figure is available at Polymer Journal online.

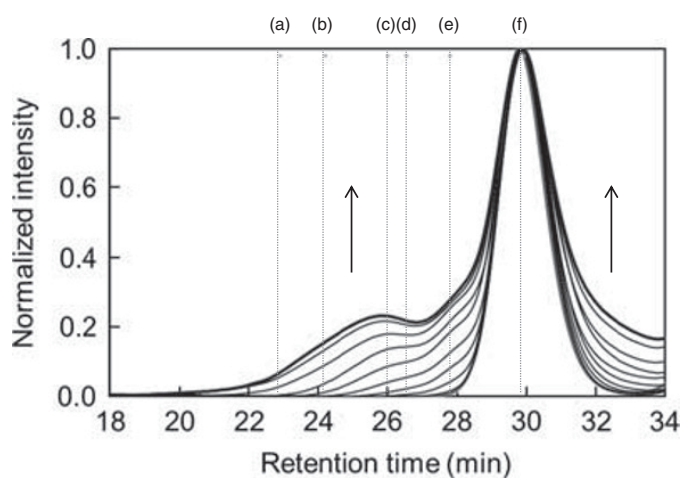

Figure 5 Gel permeation chromatographs of the immersed solution from a PS $\left(M_{\mathrm{w}}=3.35 \times 10^{3}\right)$ film (thickness $160 \mathrm{~nm}$ ) after different irradiation times $(0,10,20,30,40,50,60,70,80$ and $90 \mathrm{~min})$ at $379 \mathrm{~K}$. The charts are normalized to the original PS molecule peak intensity observed at a retention time of $29.8 \mathrm{~min}$. The vertical dotted reference lines correspond to the following PS molecular weights: (a) $M_{\mathrm{w}}=6.5 \times 10^{4}$; (b) $\quad M_{\mathrm{w}}=3.0 \times 10^{4} ; \quad$ (c) $\quad M_{\mathrm{w}}=1.34 \times 10^{4} ; \quad$ (d) $\quad M_{\mathrm{w}}=1.07 \times 10^{4}$; (e) $M_{\mathrm{w}}=6.93 \times 10^{3}$ and (f) $M_{\mathrm{w}}=3.35 \times 10^{3}$. A full color version of this figure is available at Polymer Journal online.

irradiation for $>90$ min show vestigial relief structures whose heights increase with irradiation time (Figure 3; also Supplementary Figures $\mathrm{S} 4 \mathrm{c}$ and d). This result suggests that PS is crosslinked and fixed at the irradiated regions.

From these results, we propose that the driving force of mass transport is the difference between the diffusibilities of original PS at the shaded region and the photoreacted PS at the irradiated region. ${ }^{19,67-69}$ Irradiated PS molecules undergo photooxidation and dimerization, which suppresses mobility relative to the original PS molecules. In fact, we observed a slight but definite increase in $T_{\mathrm{g}}$ after irradiation (Supplementary Figure S8). This result implies an increase in the viscosity of the PS film at the irradiated region at a constant temperature. Such a difference in diffusibility should induce a PS molecule to flow from shaded to irradiated regions. In addition, surface tension should cause SRG structural features to become sinusoidal. 

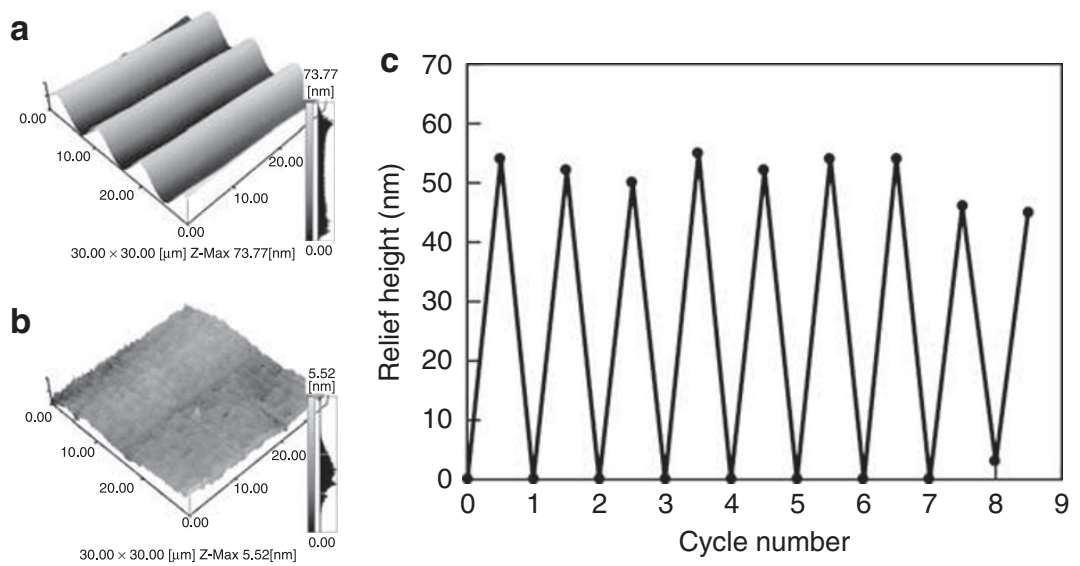

d Condition for writing and erasing the SRG

\begin{tabular}{lccccccccc}
\hline Cycle number & 1 & 2 & 3 & 4 & 5 & 6 & 7 & 8 & 9 \\
\hline Writing time (min) & 35 & 20 & 17 & 17 & 17 & 17 & 17 & 17 & 17 \\
Writing temperature (K) & 379 & 379 & 382 & 382 & 386 & 390 & 394 & 406 & 414 \\
Erasing time (min) & 60 & 60 & 60 & 60 & 60 & 60 & 60 & 60 & - \\
Erasing temperature (K) & 396 & 400 & 404 & 408 & 416 & 427 & 431 & 455 & - \\
\hline
\end{tabular}

Figure 6 Reversible SRG formation on a PS $\left(M_{\mathrm{w}}=3.35 \times 10^{3}\right.$ ) film (thickness $160 \mathrm{~nm}$ ): (a) AFM image after the sixth writing process by UV-light irradiation through a photomask for $17 \mathrm{~min}$ at $390 \mathrm{~K}$; (b) AFM image after the sixth erasing process by heating at $427 \mathrm{~K}$ for $1 \mathrm{~h}$; (c) Plot of SRG relief height as a function of cycle number during reversible SRG formation and (d) SRG writing and erasing conditions for (c). A full color version of this figure is available at Polymer Journal online.

\section{Reversibility of SRG formation}

As mentioned in the Introduction, an important advantage of a photoinduced SRG system as a micro-manufacturing method is that an SRG can be erased by heating and then reconstructed by irradiation with patterned light. We investigated the possibility of such reversibility in our SRG system. The possibility of erasing the SRG structure was first investigated. We found that the SRG structure formed on the PS film can be erased by heating but not by uniform UV light irradiation, just as we had observed and reported previously for amorphous spirooxazine molecular film. ${ }^{19}$ The degree of photoreaction determines whether SRG can be erased (Supplementary Figure S9). Although the SRG structure before crosslinking was erased rapidly, the SRG structure after crosslinking could not be erased completely by heating. Thus, the SRG structure was produced by short-time irradiation time so that crosslinking might not proceed in reversible SRG formation experiments.

The SRG structure can then be reconstructed on the film in a manner similar to that of its first photoinscription. Figures $6 a$ and $b$ show AFM images taken after the sixth cycle of patterned UV light irradiation and heating of the PS film. Figure $6 \mathrm{c}$ shows a plot of SRG relief height as a function of cycle number; reversible SRG formation can apparently be performed for at least seven cycles with a constant amplitude of $\sim 50 \mathrm{~nm}$. Figure $6 \mathrm{~d}$ lists the required conditions for successful writing and erasing. Here, the writing time was shortened according to the repeated cycles to obtain a constant amplitude, because the growth of SRG was slow in the initial stage as shown in Figure 3. The temperature during both writing and erasing must be increased over the course of the repeated cycles (for writing, from 379 to $414 \mathrm{~K}$; for erasing, from 396 to $455 \mathrm{~K}$ ). However, a completely flat surface could not be obtained after the eighth heating process. These results indicate that randomization of the photoreactant by heating allows the film to initialize a photogenerated SRG only as long as photopolymerization does not proceed to a significant extent.

\section{CONCLUSION}

In conclusion, we have succeeded in reversible SRG formation using general purpose PS films by repeated processes of spatially patterned UV light irradiation and heating. When a film is irradiated with spatially patterned UV light that can excite the electronic state of PS, surface relief structures are produced because of lateral material transfer from shaded to irradiated areas. The efficiency of SRG formation heavily depends on the temperature of the film; SRG structures are formed efficiently at temperatures $\sim 20 \mathrm{~K}$ higher than the bulk glass transition temperature. SRG growth is synchronized with PS photoreaction, including photooxidation and dimerization. Thus, we propose that surface relief structures are formed because of the difference between the diffusibilities of the irradiated photoreacted PS and the shaded original PS. This SRG formation method can be applied to PS of various molecular weights and generalized to different polymer systems.

\section{ACKNOWLEDGEMENTS}

We thank Enago (http://www.enago.jp) for the English language review. This work was supported in part by a Grant-in-Aid for Scientific Research (23750153) from the Ministry of Education, Culture, Sports, Science and Technology and the Foundation for the Promotion of Material Science and Technology of Japan (MST Foundation). 
1 Ito, T. \& Okazaki, S. Pushing the limits of lithography. Nature 406, 1027-1031 (2000).

2 Bratton, D., Yang, D., Dai, J. \& Ober, C. K. Recent progress in high resolution lithography. Polym. Adv. Technol. 17, 94-103 (2006).

3 Geissler, M. \& Xia, Y. Patterning: principles and some new developments. Adv. Mater. 16, 1249-1269 (2004).

4 Nie, Z. \& Kumacheva, E. Patterning surfaces with functional polymers. Nat. Mater. 7, 277-290 (2008).

5 Photoreactive Organic Thin Films. (eds. Sekkat, Z. \& Knoll, W.) (Academic Press, San Diego, 2002).

6 Viswanathan, N. K., Kim, D. Y., Bian, S., Williams, J., Liu, W., Li, L., Samuelson, L., Kumar, J. \& Tripathy, S. K. Surface relief structures on azo polymer films. J. Mater Chem. 9, 1941-1955 (1999).

7 Delaire, J. A. \& Nakatani, K. Linear and nonlinear optical properties of photochromic molecules and materials. Chem. Rev. 100, 1817-1845 (2000).

8 Yager, K. G. \& Barrett, C. J. All-optical patterning of azo polymer films. Curr. Opin. Solid State Mater. Sci. 5, 487-494 (2001).

9 Natansohn, A. \& Rochon, P. Photoinduced motions in azo-containing polymers. Chem. Rev. 102, 4139-4175 (2002).

10 Seki, T. Photoresponsive self-assembly motions in polymer thin films. Curr. Opin. Solid State Mater. Sci. 10, 241-248 (2006).

11 Fukuda, T., Matsuda, H., Shiraga, T., Kimura, T., Kato, M., Viswanathan, N. K., Kumar, J. \& Tripathy, S. K. Photofabrication of surface relief grating on films of azobenzene polymer with different dye functionalization. Macromolecules 33 , 4220-4225 (2000).

12 Ubukata, T., Isoshima, T. \& Hara, M. Wavelength-programmable organic distributedfeedback laser based on a photoassisted polymer-migration system. Adv. Mater. 17, 1630-1633 (2005).

13 Harada, K., Itoh, M., Yatagai, T. \& Kamemaru, S. Application of surface relief hologram using azobenzene containing polymer film. Opt. Rev. 12, 130-134 (2005).

14 Ishow, E., Brosseau, A., Clavier, G., Nakatani, K., Pansu, R. B., Vachon, J. -J., Tauc, P., Chauvat, D., Mendonça, C. R. \& Piovesan, E. Two-photon fluorescent holographic rewritable micropatterning. J. Am. Chem. Soc. 129, 8970-8971 (2007).

15 Zettsu, N., Ogasawara, T., Mizoshita, N., Nagano, S. \& Seki, T. Photo-triggered surface relief grating formation in supramolecular liquid crystalline polymer systems with detachable azobenzene unit. Adv. Mater. 20, 516-521 (2008).

16 Goldenberg, L. M., Kulikovsky, L., Kulikovska, O. \& Stumpe, J. New materials with detachable azobenzene: effective, colourless and extremely stable surface relief gratings. J. Mater. Chem. 19, 8068-8071 (2009).

17 Ubukata, T., Takahashi, K. \& Yokoyama, Y. Photoinduced surface relief structures formed on polymer films doped with photochromic spiropyrans. J. Phys. Org. Chem. 20, 981-984 (2007)

18 Ubukata, T., Yamaguchi, S. \& Yokoyama, Y. Photoinduced surface relief structures formed on polymer films mixed with diarylethenes. Chem. Lett. 36, 1224-1225 (2007).

19 Ubukata, T., Fujii, S. \& Yokoyama, Y. Reversible phototriggered micromanufacturing using amorphous photoresponsive spirooxazine film. J. Mater. Chem. 19, 3373-3377 (2009).

20 Kikuchi, A., Harada, Y., Yagi, M., Ubukata, T., Yokoyama, Y. \& Abe, J. Photoinduced diffusive mass transfer in $0-\mathrm{Cl}-\mathrm{HABI}$ amorphous thin films. Chem. Commun. 46, 2262-2264 (2010).

21 Tsuboi, Y., Sakashita, S., Hatanaka, K., Fukumura, H. \& Masuhara, H. Phototherma ablation of polystyrene film by $248 \mathrm{~nm}$ excimer laser irradiation: a mechanistic study by time-resolved measurements. Laser Chem. 16, 167-177 (1996).

22 Srinivasan, R. \& Leigh, W. J. Ablative photodecomposition: action of far-ultraviolet $(193 \mathrm{~nm})$ laser radiation on poly(ethylene terephthalate) films. J. Am. Chem. Soc. 104, 6784-6785 (1982)

23 Kawamura, Y., Toyoda, K. \& Namba, S. Effective deep ultraviolet photoetching of polymethyl methacrylate by an eximer laser. Appl. Phys. Lett. 40, 374-375 (1982).

24 Brannon, J. H., Lankard, J. R., Baise, A. I., Burns, F. \& Kaufman, J. Excimer laser etching of polyimide. J. App. Phys. 58, 2036-2043 (1985).

25 Srinivasan, R., Braren, B. \& Dreyfus, R. W. Ultraviolet laser ablation of polyimide films J. Appl. Phys. 61, 372-376 (1987).

26 Srinivasan, R., Braren, B. \& Casey, K. G. Nature of 'incubation pulses' in the ultraviolet laser ablation of polymethyl methacrylate. J. Appl. Phys. 68, 1842-1847 (1990).

27 Park, J. -H., Yoon, T. -Y., Lee, W. -J. \& Lee, S. -D. Multi-domain liquid crystal display with self-aligned 4-domains on surface relief gratings of photopolymer. Mol. Cryst. Liq. Cryst. 375, 433-440 (2002).

28 Zhou, J., Sun, C., Xiong, B., Wang, J. \& Luo, Y. Polymer gratings with low surface relief based on photopolymerization-induced internal diffusion. Appl. Phys. Lett. 84, 30193021 (2004).

29 Ramanujam, P. S. \& Berg, R. H. Photodimerization in dipeptides for high capacity optical digital storage. Appl. Phys. Lett. 85, 1665-1667 (2004).

30 Yamaki, S., Nakagawa, M., Morino, S. \& Ichimura, K. Surface relief gratings generated by a photocrosslinkable polymer with styrylpyridine side chains. Appl. Phys. Lett. 76, 2520-2522 (2000).

31 Ono, H., Emoto, A., Kawatsuki, N. \& Hasegawa, T. Self-organized phase gratings in photoreactive polymer liquid crystals. Appl. Phys. Lett. 82, 1359-1361 (2003).
32 Kawatsuki, N., Hasegawa, T., Ono, H. \& Tamoto, T. Formation of polarization gratings and surface relief gratings in photocrosslinkable polymer liquid crystals by polarization holography. Adv. Mater. 15, 991-994 (2003).

33 Ono, H., Hatayama, A., Emoto, A. \& Kawatsuki, N. Migration induced reorientation and anisotropic grating formation in photoreactive polymer liquid crystals. Opt. Mater. 30, 248-254 (2007)

34 Kawatsuki, N., Tashima, A., Manabe, S., Kondo, M., Okada, M., Matsui, S., Emoto, A. \& Ono, H. Holographic recording in a photo-cross-linkable liquid crystalline copolymer using a 325-nm laser with various polarizations. React. Funct. Polym. 70, 980-985 (2010).

35 Kawatsuki, N., Kondo, M., Okada, M., Matsui, S., Ono, H. \& Emoto, A. Surface relief formation in photo-cross-linkable polymer/benzophenone composite films using $325 \mathrm{~nm}$ laser. Jap. J. Appl. Phys. 49, 08207 (2010).

36 de Sarkar, M., Gill, N. A., Whitehead, J. A. \& Crawford, G. P. Effect of monomer functionality on the morphology and performance of the holographic transmission gratings recorded on polymer dispersed liquid crystals. Macromolecules 36, 630-638 (2003).

37 Mazzulla, A., Pagliusi, P., Provenzano, C., Russo, G., Carbone, G. \& Cipparrone, G. Surface relief gratings on polymer dispersed liquid crystals by polarization holography. Appl. Phys. Lett. 85, 2505-2507 (2004).

38 Leewis, C. M, de Jong, A. M., van IJzendoorn, L. J. \& Broer, D. J. Reaction-diffusion model for the preparation of polymer gratings by patterned ultraviolet illumination. J. Appl. Phys. 95, 4125-4139 (2004).

39 Leewis, C. M., de Jong, A. M., van IJzendoorn, L. J. \& Broer, D. J. Simulations with a dynamic reaction-diffusion model of the polymer grating preparation by patterned ultraviolet illumination. J. Appl. Phys. 95, 8352-8356 (2004).

40 Sánchez, C. de Gans, B-J., Kozodaev, D., Alexeev, A., Escuti, M. J., van Heesch, C. Bel, T., Schubert, U. S., Bastiaansen, C. W. M. \& Broer, D. J. Photoembossing of periodic relief structures using polymerization-induced diffusion: a combinatorial study. Adv. Mater. 17, 2567-2571 (2005).

41 Hermans, K., Wolf, F. K., Perelaer, J., Janssen, R. A. J., Schubert, U. S., Bastiaansen, C. W. M. \& Broer, D. J. High aspect ratio surface relief structures by photoembossing. Appl. Phys. Lett. 91, 174103 (2007).

42 Goldenberg, L., Sakhno, 0. \& Stumpe, J. Application of Norland adhesive for holographic recording. Opt. Mater. 27, 1379-1385 (2005)

43 Aoki, K. \& Ichimura, K. Self-developable surface relief photoimaging generated by anionic UV-curing of epoxy resins. Polym. J. 41, 988-992 (2009).

44 Zhao, D., Xu, Z., Wang, G., Cao, H., Li, W., He, W., Huang, W., Yang, Z. \& Yang, H. Formation of surface relief gratings with homeotropically oriented photopolymer from a photocross-linkable organic monomer. Phys. Chem. Chem. Phys. 12, 1436-1439 (2010).

45 Baldus, O., Leopold, A., Hagen, R., Bieringer, T. \& Zilker, S. J. Surface relief gratings generated by pulsed holography: a simple way to polymer nanostructures without isomerizing side-chains. J. Chem. Phys. 114, 1344-1349 (2001).

46 Ramanujam, P. S. Optical fabrication of nano-structured biopolymer surfaces. Opt. Mater. 27, 1175-1177 (2005).

47 Krebs, F. C. \& Ramanujam, P. S. Holographic recording in a series of conjugated polymers. Opt. Mater. 28, 350-354 (2006).

48 Kaganovskii, Y., Beke, D. L. \& Kökényesi, S. Kinetics of photoinduced surface patterning in chalcogenide thin films. Appl. Phys. Lett. 97, 061906 (2010).

49 Ubukata, T., Higuchi, T., Zettsu, N., Seki, T. \& Hara, M. Spontaneous motion observed in highly sensitive surface relief formation system. Colloids Surf. A 257-258, 123-126 (2005).

50 Grassie, N. \& Weir, N. A. The photooxidation of polymers. I. Experimental methods. J. Appl. Polym. Sci. 9, 963-974 (1965).

51 Grassie, N. \& Weir, N. A. The photooxidation of polymers. II. Photolysis of polystyrene. J. Appl. Polym. Sci. 9, 975-986 (1965).

52 Grassie, N. \& Weir, N. A. The photooxidation of polymers. III. Photooxidation of polystyrene. J. Appl. Polym. Sci. 9, 987-998 (1965).

53 Grassie, N. \& Weir, N. A. The photooxidation of polymers. IV. A note on the coloration of polystyrene. J. Appl. Polym Sci. 9, 999-1003 (1965).

54 Geuskens, G., Baeyens-Volant, D., Delaunois, G., Lu-Vinh, Q., Piret, W. \& David, C. Photo-oxidation of polymers-I: a quantitative study of the chemical reactions resulting from irradiation of polystyrene at $253.7 \mathrm{~nm}$ in the presence of oxygen. Eur. Polym. J. 14, 291-297 (1978).

55 Geuskens, G., Baeyens-Volant, D., Delaunois, G., Lu-Vinh, Q., Piret, W. \& David, C. Photo-oxidation of polymers-II: the sensitized decomposition of hydroperoxides as the main path for initiation of the photo-oxidation of polystyrene irradiated at $253.7 \mathrm{~nm}$. Eur. Polym. J. 14, 299-303 (1978).

56 David, C., Baeyens-Volant, D., Delaunois, G., Lu-Vinh, Q., Piret, W. \& Geuskens, G. Photo-oxidation of polymers-III: molecular weight changes in the photolysis and photo-oxidation of polystyrene. Eur. Polym. J. 14, 501-507 (1978).

57 Geuskens, G. The photooxidation of polystyrene. In Developments in Polymer Degradation (ed. Grassie, N.) vol. 3 207-227 (Applied Science, London, 1981).

58 Barada, D., Itoh, M. \& Yatagai, T. Computer simulation of photoinduced mass transport on azobenzene polymer films by particle method. J. Appl. Phys. 96, 4204-4210 (2004).

59 Barada, D., Fukuda, T., Itoh, M. \& Yatagai, T. Proposal of novel model for photoinduced mass transport and numerical analysis by electromagnetic-induced particle transport method. Jap. J. Appl. Phys. 45, 465-469 (2006).

60 Nakano, H., Tanino, T., Takahashi, T., Ando, H. \& Shirota, Y. Relationship between molecular structure and photoinduced surface relief grating formation using 
azobenzene-based photochromic amorphous molecular materials. J. Mater. Chem. 18, 242-246 (2008).

61 Millan, M. D., Locklin, J., Fulghum, T., Baba, A. \& Advincula, R. C. Polymer thin film photodegradation and photochemical crosslinking: FT-IR imaging, evanescent waveguide spectroscopy, and QCM investigations. Polymer 46, 5556-5568 (2005).

62 Lucas, P. C. \& Porter, R. S. On the fate of the phenyl ring in polystyrene photooxidation. Macromolecules 27, 3666-3668 (1994).

63 Wells, R. K., Royston, A \& Badyal, J. P. S. Direct evidence for the generation of phenyl radicals and crosslinking during the photolysis of a polystyrene film. Macromolecules 27, 7465-7468 (1994).

64 Kuzina, S. I. \& Mikhailov, A. I. Photochain destruction of excited peroxide polystyrene macroradicals. J. Photochem. Photobiol. A 163, 367-372 (2004).
65 Mailhot, B. \& Gardette, J. -L. Polystyrene photooxidation. 1. Identification of the IRabsorbing photoproducts formed at short and long wavelengths. Macromolecules 25, 4119-4126 (1992).

66 Mailhot, B. \& Gardette, J. -L. Polystyrene photooxidation. 2. A pseudo wavelength effect. Macromolecules 25, 4127-4133 (1992).

67 Lefin, P., Fiorini, C. \& Nunzi, J. -M. Anisotropy of the photo-induced translation diffusion of azobenzene dyes in polymer matrices. Pure. Appl. Opt. 7, 71-82 (1998).

68 Frank, B., Gast, A. P., Russell, T. P., Brown, H. R. \& Hawker, C. Polymer mobility in thin films. Macromolecules 29, 6531-6534 (1996).

69 Karapanagiotis, I. \& Gerberich, W. W. Curvature driven flow of thin polymer films and diffusivity measurements. Macromolecules 38, 3420-3425 (2005).

Supplementary Information accompanies the paper on Polymer Journal website (http://www.nature.com/pj) 